\title{
How does culture affect individual adaptation to climate-driven floods?
}

\author{
Brayton Noll 1, a, Tatiana Filatova ${ }^{1,2}$, and Ariana Need 3 \\ ${ }^{1}$ Department of Governance and Technology for Sustainability, University of Twente, Drienerlolaan 5, 7522 NB \\ Enschede, the Netherlands \\ ${ }^{2}$ School of Information, Systems and Modeling, Faculty of Engineering and IT, University of Technology Sydney, \\ 15 Broadway, Ultimo NSW 2007, Australia \\ ${ }^{3}$ Department of Public Administration, University of Twente, Drienerlolaan 5, 7522 NB Enschede, the Netherlands
}

\begin{abstract}
Expanding human developments and climate change have exacerbated the impact of natural hazards worldwide. In particular, accelerating flood risks entails that more traditional top down measures are complimented by individual adaptation at the household level. Worldwide, social surveys provide rich information about factors motivating individual adaptation to climate-driven floods. Notably, much of this empirical research has focused on the Global North. Yet, empirical research explicitly measuring whether and how climate change adaptation varies across cultures is lacking. Using Hofstede's Cultural Rankings as a measure of national culture, we utilize meta-regression analysis to investigate the effects of culture on individuals' motivation to adapt to climate-driven floods. This research makes a unique contribution to the literature by providing some of the first empirical evidence on the interaction effects between culture and individual flooding adaptation motivation based on the data collected across a large sample of countries. The results provide strong statistical support for the importance of considering cultural differences when empirically assessing limits of individual adaptation worldwide.
\end{abstract}

\section{Introduction}

Adaptation to climate change is at the forefront of both political and academic environmental discourse (Brown et al., 2019, Conway et al., 2019).). Recent cross-national studies on climate change perception and public adaptation explicitly highlight the need to consider cultural and geographical differences when looking at individual perception and adaptation to climate change across countries (Poortinga et al., 2019). When considering climate change impacts, floods are among the most devastating and costly (Aerts et al., 2014). Similar to the broader field of climate change adaptation, there is strong agreement that culture influences natural flood risk perception and individual adaptation behavior (Kruger et al., 2015).

However, with a few exceptions, cross-national empirical research has been limited; both in terms of the number of publications and in the number of countries included in the surveys. Methods to analyze culture often require ethnographic and observational research and yield qualitative data that is more frequently utilized by anthropologists and sociologists, rather than climate and disaster researchers (Adger et al., 2013; Cannon, 2015). There are however several approaches that quantitatively characterize different national cultural dimensions. For the

a Corresponding author: b.1.noll@utwente.nl

DOI 10.3311/FLOODRisk2020.4.5 purposes of this paper we selected Hofstede's Rankings for its suggested superior ability to predict behavioral frequencies when compared to Globe (GLOBE, 2019) (Smith et al., 2006) and for the greater data availability for countries in which flood surveys were conducted when compared to the World's Value Survey rankings (Inglehart et al., 2014).

The aim of this paper is to quantitatively and systematically examine if there are observable patterns for the different factors motivating individual climate change adaptation behavior that can be explained by national culture. To test this, we review 53 independent studies and collect bi-variate associations of factors motivating individual adaptation. Based on the country where the survey data was conducted, we plot the collected bi-variate associations against Hofstede's national cultural rankings.

The innovative contribution of this paper to the literature is two-fold. First, to the best of our knowledge, for the first time the interaction effects between culture and factors motivating private climate change adaption are measured with a sufficiently large country sample - more than 10 countries (Hofstede et al., 2010, p.30) - to distinguish cultural differences. Second, our extensive meta-analytic review affords statistical support for a previously contradicted difference in the effect size of risk perception toward intended vs. undergone adaptation. This 
Factors Motivating Adaptation

\begin{tabular}{|c|l|}
\hline Risk Perception (RP) & $\begin{array}{l}\text { All reported ES for Risk Perception are included here. Combines ES for intended and } \\
\text { undergone adaptation and ES probability and damage }\end{array}$ \\
\hline RP: Undergone Adaptation & Combines both ES for probability and damage given that adaptation has already occurred \\
\hline RP: Intended Adaption & $\begin{array}{l}\text { Combines both ES for probability and damage given a reported intention to pursue } \\
\text { adaptation }\end{array}$ \\
\hline RP: Probability of flood & $\begin{array}{l}\text { Combines the ES of individual assessment of probability of flooding in } \\
\text { both intended and undergone adaptation }\end{array}$ \\
\hline RP: Damage due to flood & $\begin{array}{l}\text { Combines the ES of individual assessment of damage from flooding in both intended and } \\
\text { undergone adaptation }\end{array}$ \\
\hline (Prior) Flood Experience & ES were collected for any mention of flood experience \\
\hline Age & ES were not collected if the ES of age was reported categorically \\
\hline Self-Efficacy (SE) & ES were collected or transformed for the Female gender \\
\hline SE: Undergone Adaptation & $\begin{array}{l}\text { All reported ES for Self Efficacy are included here. Combines ES for intended and } \\
\text { undergone adaptation }\end{array}$ \\
\hline ES: Intended Adaptation & ES indicates relationship between Self Efficacy and undergone adaptation \\
\hline Social Influence & $\begin{array}{l}\text { 'Information received', 'expectations', 'social support', and 'perceived stigma(s)' from } \\
\text { family, friends, neighbors, and/or the local community) }\end{array}$ \\
\hline Institutional Faith & $\begin{array}{l}\text { 'Information' provided by a governmental body/ the media, and/or the individual's 'trust' } \\
\text { in the government, and/or 'adaptive actions' undertaken by a governmental body }\end{array}$ \\
\hline
\end{tabular}

Table 2. The 13 factors motivating private adaptation to floods used in our meta-analysis.

distinction between actual and intended adaptation to climate-induced risks is important in understanding the feedback effects between individual behavior and perceptions.

The remainder of the paper is organized as follows: Section 2 outlines the methods used to collect and transform the data. In Section 3 we test for a difference in factors motivating intended vs. undergone adaptation to floods, sample size permitting. Following this, we present the meta-regression analysis to measure the interaction effects between factors motivating private adaptation and Hofstede's six cultural rankings. Section 4 discusses the implications of this research and concludes.

\section{Methods}

\subsection{Literature Search Strategy}

To obtain data on individual behavior and flooding in a multinational context, we conducted a thorough literature search in 2019. Six different keyword combinations of "Individual", "Household", "Flood", "Adaptation", "Protection", "Motivation", and "Survey" were searched on SCOPUS and Web of Science, and Google Scholar. If the title or abstract mentioned a survey, a sample size, empirical data, or a factor motivating adaptation, the article was screened to determine if it contained bi-variate associations or effect sizes (ES) of factors motivating private flooding adaptation. ES are a way to measure the strength of an association and once standardized, allow for cross-study comparisons of the effects that different factors have on adaptation motivation. To enable meta-regression analysis, if 10 or more surveys reported a factor that influences adaptation, we included it in our analysis. Ultimately the aforementioned criteria yielded selected seven factors that were reported sufficiently frequent. Two factors: 'Risk Perception' and 'Self Efficacy' were asked often enough that we were able to distinguish between their effect in motivating intended, vs. undergone adaptation. Additionally, for Risk Perception, whenever possible we distinguished between its two components by recording whether it was the probability of the flood or the expected damage of the flood that motivated adaptation. These subcategories led to a total of 13 factors that motivate flooding adaptation (Table 1).

Focusing on these 13 factors, we narrowed down our sample of surveys on private adaptation to climate driven flooding to a total of 72 reported surveys. If a study did not measure an adaptive action for protecting one's current home and only measured evacuation or out migration, it was excluded; as this is a fundamentally different type of adaptation (Koerth et al. 2017). Further, if the study reported a combined result for multiple countries, or did not reported standardized ES, it was excluded. To maximize the number of data points, we used the supplemental flooding adaptation motivation data provided by Van Valkengoed and Steg (2019) that they received by contacted authors for data not available in the original publications. Following these search criteria, we compiled a dataset based on 53 independent publications or studies (56 surveys) from 25 different countries, and the total number of respondents $\mathrm{N}=38,891$.

\subsection{Cultural Rankings}

The cultural ranking scores for each of Hofstede's cultural dimensions are based on the empirical data collected from internationally comparable surveys 
(Hofstede et al., 2010). Namely, we use six cultural dimensions in our analysis:

\author{
I. Individualism vs. Collectivism \\ II. High Power Distance vs. Low Power Distance \\ III. High Uncertainty Avoidance vs. Low \\ Uncertainty Avoidance \\ IV. Masculinity vs. Femininity \\ V. Long Term Orientation vs. Short Term \\ Orientation \\ VI. Indulgence vs. Restraint
}

Within each of these six dimensions, a country can score between 1 - 100, relative to other countries. A higher score indicates more of the core characteristic highlighted in bold above. All of the cultural ranking scores were taken from Hofstede (2019) in January 2020 with two exceptions: Ethiopia did not have scores for two cultural dimensions: Long Term Orientation and Indulgence; and was excluded from the analysis in only these two categories. Cambodia has no scores for any cultural dimension, so we calculated coefficient estimates using a recent article (Berkvens, 2017) that discusses Hofstede's cultural dimensions in Cambodia's ranked neighbors.

\subsection{Data Processing: Effect Sizes}

To allow comparison between the collected ES of the 13 factors motivating individual adaptation with the six cultural dimensions, initially we needed to standardized them. First, we transformed all ES into Pearson's r, and then converted them using the variance-stabilizing Fisher's $r$ to $z$ transformation (Borenstein et al., 2009). Second, once transformed, we applied the random effects model on the factors in the dataset, weighted by the inverse variance, within and between the surveys. A random effects model, as opposed to a fixed effect model reflects a belief that there is more than one "true" ES. Based on the findings of Veroniki et al. (2016), we selected PauleMandel's estimator for calculating the between study variance. The random-effects-weights for the individual and pooled values were calculated in $\mathrm{R}$ (3.6.1) using the "Metafor" package (Viechtbauer, 2015). We applied the assigned weights (percentages) to each study, then multiplied by the number of studies that reported an ES for a given factor to have the appropriate random effect weights for each individual study. This permitted us to run analysis with the individual studies weighted by random effects (e.g. meta-regression analysis) and not simply consider the pooled effect size. Third, the sum of the weighted individual study values was used to cross-check the pooled random-effect-weighted means. Finally, the weighted z-transformed correlations were re-converted into the commonly used ES of Pearson's $r$ for the analysis with culture and the reporting on meta-analytic findings. Figure 2 illustrates the pooled random-effect-weighted of the seven main ES in Pearson's r.

It is valuable however, to consider the difference in effect sizes that different factors have in motivating intended adaptation vs. undergone or concurrent action.
Our collected sample size enabled testing of this difference with two variables: Risk Perception and Self Efficacy, using Bayes factor, we present our findings in the following section. We could not do the same with Flood Experience (the other factor with a relatively large N) due to a lack of studies that measured intended adaptation and reported on the ES of Flood Experience.

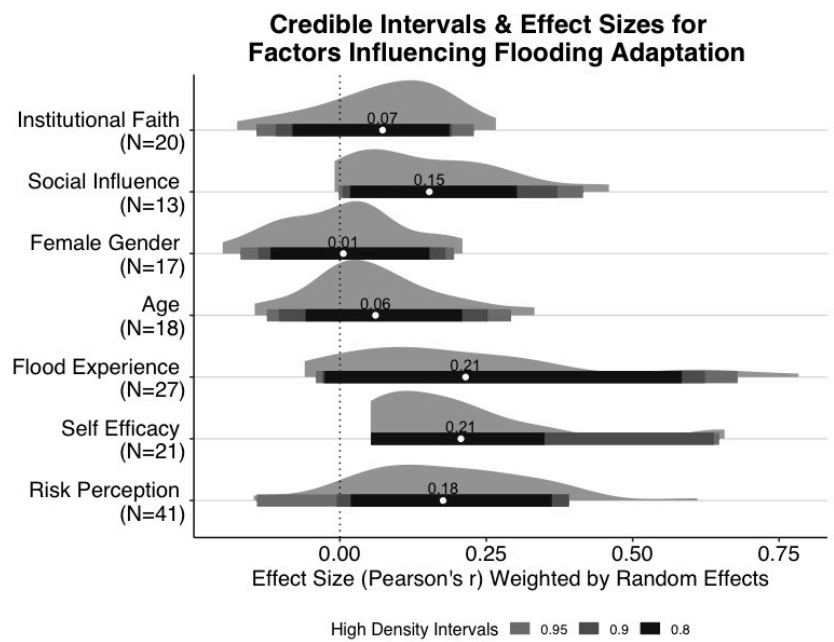

Figure 1. The Effect Sizes for the 7 primary collected factors motivating flood adaptation (both intention and undergone action grouped together) after weighting by random effects.

\section{Results}

\subsection{Intention vs. Action}

Acknowledging the potential feedback from previously undergone adaptive actions on individual risk perception is important for understanding individual adaptation motivation. Previously, Bamberg et al. (2017) used meta-analytic data collected from PMT surveys and found that the effect of Risk Perception on undergone action is generally higher than the effect on intended adaptation. This finding contradicts the hypothesis of Bubeck et al. (2012) that individual risk perception should diminish after one has taken a flood adaptation measure. The dataset behind our meta-analysis utilizes a larger sample of studies compared to Bamberg et al. (2017)'s and goes beyond studies guided by PMT alone. We compare the mean ES of Risk Perception toward intended private adaptation with the mean ES of Risk Perception after households have already undertaken an adaptive action (Figure 2).

Bayes' Factor of 16.47 is a "strong" indication that the ES for Risk Perception is greater for intended adaptation to floods than it is for already undergone or concurrent action Jeffreys (1998). This suggests there are cases where households may begin to feel sufficiently protected and ignore flood risk after adaptation actions have been taken. Importantly, in contrast to the previous meta-analysis (Bamberg et al., 2017), our analysis supports the original assumption of (Bubeck et al., 2012) regarding feedbacks between adaptation to floods and individual risk perception unfolding over time. We find no 
statistically significant difference between Self Efficacy and intended vs. undergone adaptation.

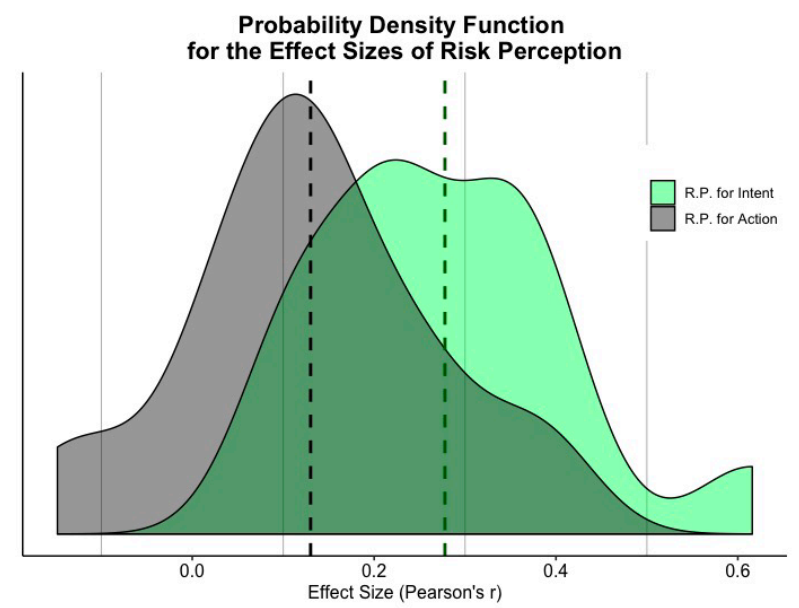

Figure 2. Probability density functions for the effect size of risk perception in influencing intention to adapt and undergone adaptation. The mean value for effect size of risk perception toward intent is: $0.28(\mathrm{~N}=14$ surveys) and for (undergone) action is: $0.13(\mathrm{~N}=30$ surveys $)$.

\subsection{Private Adaptation and Cultural Dimensions}

To empirically test if there are observable patterns for different factors motivating individual adaptation that can be explained by national culture, we use Hofstede's six cultural rankings. We plot the converted ES of factors motivating individual adaptation against the cultural rankings based on the country where the survey was conducted. Table 2 presents the results.

\subsection{Flood Experience and Culture}

How an individual experience a natural hazard and the manner in which a society prepares, is impacted, and recovers from an event is strongly influenced by aspects of culture (Kruger et al., 2015). One example of a factor influenced by culture is prior flood experience. In general, personal experience with a flood is a strong indicator of future adaptation (Wachinger et al., 2013). Our results indeed support this idea; however, the magnitude of the effect appears to be mediated by culture. Several cultural dimensions: Individualism, Power Distance and Uncertainty Avoidance have significant linear relationships with prior Flood Experience's influence on adaptation (Figure 3).

The Individualism dimension is the most highly correlated to the ES of flood experience motivating adaptation, is significant after the Bonferroni correction, and explains the most variance in a linear model. In individualistic societies the 'group' an individual is responsible for, and socially answers to, is smaller than in more collectivist ones. Additionally, in individualistic societies, public areas are less frequently utilized for family and social gatherings (Hofstede et al., 2010;

\begin{tabular}{|c|c|c|c|c|c|c|}
\hline & \multicolumn{6}{|c|}{ Hofstede's Cultural Rankings } \\
\hline & $\mathbf{I}$ & II & III & IV & $\mathbf{V}$ & VI \\
\hline \multicolumn{7}{|l|}{$\begin{array}{l}\text { Risk Perception (RP) } \\
(\mathrm{N}=41, \mathrm{n}=26856, \mathrm{c}=16)\end{array}$} \\
\hline \multicolumn{7}{|l|}{$\begin{array}{l}\text { RP: Undergone adapt. } \\
(\mathrm{N}=30, \mathrm{n}=21954, \mathrm{c}=13)\end{array}$} \\
\hline \multicolumn{7}{|l|}{$\begin{array}{l}\text { RP: Intended adapt. } \\
(\mathrm{N}=14, \mathrm{n}=5182, \mathrm{c}=7)\end{array}$} \\
\hline $\begin{array}{l}\text { RP: Probability } \\
(\mathrm{N}=15, \mathrm{n}=7082, \mathrm{c}=10)\end{array}$ & & & & $-0.003 *$ & & $0.007 * *$ \\
\hline $\begin{array}{l}\text { RP: Damage } \\
(\mathrm{N}=15, \mathrm{n}=5626, \mathrm{c}=11)\end{array}$ & & & & & $-0.006^{*}$ & \\
\hline $\begin{array}{l}\text { Flood Experience } \\
(\mathrm{N}=27, \mathrm{n}=18257, \mathrm{c}=16)\end{array}$ & $\underline{-0.005}^{* * *}$ & $0.006^{* *}$ & $-0.004 *$ & & & \\
\hline $\begin{array}{l}\text { Age } \\
(\mathrm{N}=18, \mathrm{n}=14294, \mathrm{c}=15)\end{array}$ & & $0.002 *$ & & & & \\
\hline $\begin{array}{l}\text { Gender (Female) } \\
(\mathrm{N}=17, \mathrm{n}=17870, \mathrm{c}=15)\end{array}$ & & $-0.002 *$ & & & & \\
\hline $\begin{array}{l}\text { Self Efficacy (SE) } \\
(\mathrm{N}=21, \mathrm{n}=10658, \mathrm{c}=15)\end{array}$ & $-0.003 *$ & $0.004^{*}$ & & & & \\
\hline $\begin{array}{l}\text { SE: Undergone adapt. } \\
(\mathrm{N}=14, \mathrm{n}=7290, \mathrm{c}=11)\end{array}$ & $-0.003 * *$ & & & & & \\
\hline \multicolumn{7}{|l|}{$\begin{array}{l}\text { SE: Intended adapt. } \\
(\mathrm{N}=10, \mathrm{n}=3648, \mathrm{c}=7)\end{array}$} \\
\hline \multicolumn{7}{|l|}{$\begin{array}{l}\text { Social Influence } \\
(\mathrm{N}=13, \mathrm{n}=6866, \mathrm{c}=10)\end{array}$} \\
\hline $\begin{array}{l}\text { Institutional Faith } \\
(\mathrm{N}=20, \mathrm{n}=19599, \mathrm{c}=12)\end{array}$ & & $\underline{0.004} * * *$ & & & & \\
\hline
\end{tabular}

${ }^{*} \mathrm{p}<0.1, * * \mathrm{p}<0.05, * * * \mathrm{p}<0.01$

Table 2: Random-effects weighted regression coefficient estimates of different factors motivating adaptation with a statistically significant relationship with Hofstede's Cultural Rankings: (I): Individualism - Collectivism, (II): High - Low Power Distance, (III) High - Low Uncertainty Avoidance, (IV): Masculinity - Femininity, (V): Long - Short Term Orientation, (VI): Indulgence - Restraint. $(\mathrm{N}=$ number of surveys, $\mathrm{n}=$ number of respondents, $\mathrm{c}=$ number of countries, significant values after the Bonferroni corrected significance level (0.008) are underlined) 
Triandis et al., 1986). Personal connection to a flood affected area is an important factor in determining if a flood experience will influence future adaptation (Barnett and Breakwell, 2001; Eiser et al., 2012). Hence it is possible that a smaller social circle and/or the lessened utilization of public space contribute to the negative relationship that Individualism has with the ES of Flood Experience motivating individual adaptation.

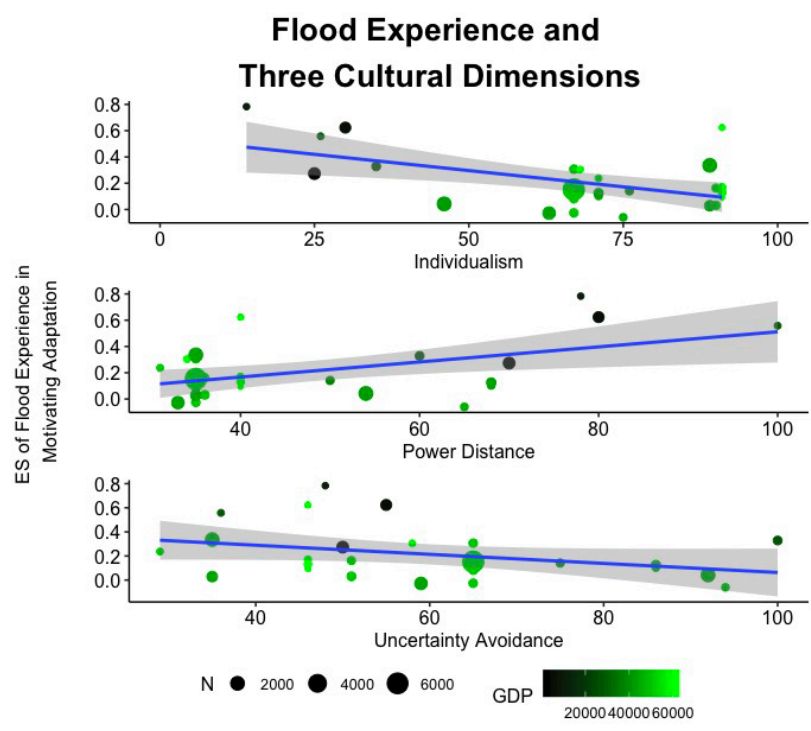

Figure 3: The effect sizes for flood experience in motivating adaptation plotted against three of Hofstede's cultural dimensions: Individualism (top), Power Distance (middle) and Uncertainty Avoidance (bottom). "N" is the size of the survey and "GDP" represents GDP per capita for the country in which the survey was conducted.

High Power Distance conversely has a positive relationship with flood experience motivating adaptation. This is expected as Power Distance and Individualism generally have an inverse relationship with one another. Furthermore, both cultural dimensions are correlated with GDP per capita (a positive relationship with Individualism and a negative one with High Power Distance). Wealth and culture are inextricably linked in many ways, especially with these two dimensions (Hofstede et al., 2010, p.108, p.132). In Table 2 the results are simple bi-variate associations. Due to the numerous links between culture and wealth and a desire to represent the direct relationship between factors motivating adaptation and cultural variables, we present the associations in the main analysis without controlling for GDP. As a robustness check, we retested the models after controlling for GDP. Almost all associations between culture and the effect sizes maintain their same level of significance.

GDP per capita however, does indeed contribute to how flood experience affects an individual. It partially represents a nation's capability to allocate resources and provide support to the communities and individuals impacted by floods (Gardoni and Murphy, 2010). These capacities could lessen the traumatic impact of a flood, and thereby contribute to prior flood experience being less of a motivating factor in countries with higher GDP per capita.
It is also likely that if a government has more resources to allocates individuals may expect to receive aid, and thus there could some incentive to 'free-ride' (Wachinger et al., 2013). Despite GDP's importance (see black-to-green gradient in Figure 3), Individualism is more highly correlated and explains slightly more variance than GDP per capita; further reinforcing the necessity of considering culture in disaster adaptation (Eiser et al., 2012; Kruger et al., 2015).

The final cultural dimension to have a significant relationship with flood experience motivating adaptation is Uncertainty Avoidance. Uncertainty Avoidance measures how averse to unknown situations members of a society are. A flood occurrence may serve as a communication vehicle, since people get an updated information on the nature of this hazard event and their vulnerability to it, as also confirmed empirically in the hedonic analysis literature (Bin and Landry, 2013). Hence, experience with a flood and the damage it brings reduces uncertainty surrounding the event. The increased clarity around flooding that follows an event, could result in a lessened adversity to flooding and explain the diminished effect that Flood Experience has in motivating adaption in societies with higher Uncertainty Avoidance. This idea is supported by (Hofstede et al., 2010, p.198) as they note that individuals from high Uncertainty Avoidance societies can paradoxically engage in risky behavior to in order to "reduce ambiguity" in their lives.

Since Flood Experience shows a statistically significant relationship with multiple cultural rankings, we select a multiple regression model to explain the most variance in the ES of Flood Experience motivating adaptation. Power Distance and Individualism cannot be in the same model due to issues with co-linearity. Thus, using stepwise model building logic, we select Individualism (the most highly correlated cultural factor) and then Uncertainty Avoidance for our model. In Equation 1 we explain the size of the effect of Flood Experience (ES $\mathrm{Exp}_{\text {) }}$ on adaptation motivation by using the cultural ranking score (C) of the two previously described cultural dimensions - Individualism and Uncertainty Avoidance with the intercept and error (e).

$$
E S_{E x p}=-0.0057\left(C_{I n d}\right)-0.0050\left(C_{U n c}\right)+0.90+e
$$

Following the data from 27 surveys from 16 countries with the total number of 18,257 respondents, the two cultural dimensions - Individualism and Uncertainty Avoidance - explain 41\% (adjusted r2, p<0.001) of the variance in the size of the effect of Flood Experience motivating adaption. With inclusion in this equation, the p-value of Uncertainty Avoidance increases its significance level to 0.05 , further suggesting its value.

\section{Discussion}

Culture is a complex, multidimensional concept that can be difficult to measure. These challenges have led researchers to frequently shy away from the inclusion or explicit consideration of culture's influence in climate 
change adaptation work (Bankoff et al., 2015; Cannon, 2015). Yet, culture directly influences many aspects of climate change adaptation motivation and is absolutely essential to include in the discourse (Adger et al., 2013). This article addresses the gap in the literature by presenting the results of meta-analysis of surveys conducted globally and reporting on individual decisions to adapt to the most devastating climate-driven hazard: flooding. Our metaanalysis of 53 independent household surveys (N:38,891) provides empirical evidence in support of considering culture when looking at individual adaptation behavior. Utilizing meta-regression with Hofstede's cultural rankings as explanatory variables, we find that national level culture indeed affects factors motivating private adaptation behavior towards flooding. For example, there is significant variation in the effect that prior Flood Experience has on adaptation motivation is explained by several cultural dimensions. The multiple-regression cultural model (utilizing Individualism and Uncertainty Avoidance) predicting the effect of prior flood experience on motivating individual adaptation, explains more than $40 \%$ of the variance in the collected effect sizes of Flood Experience affecting adaptation. This finding provides a clear incentive for researchers, modelers, and policy makers to utilize the easily accessible national level culture data available for inclusion in their work and models.

Our global analysis of surveys permits us to further investigate of the relationship between individual risk perception and intended versus undergone adaptation to flooding. In contrast to previous analysis based on a more limited sample of surveys (Bamberg et al., 2017) we find a strong difference between individual Risk Perception toward undergone and intended adaptation. The higher effect sizes in Risk Perception toward intended adaptation, compared to undergone adaptation is likely due to the feedback the concurrent action has in lowering one's perceived risk once the action is completed. Bubeck et al. (2012a) propose that longitudinal data would be a revealing method to further investigate this feedback. We agree and additionally suggest that this method would be useful in illuminating the extent to which intention leads to action. Furthermore, as demonstrated and discussed by Osberghaus (2017), longitudinal data can be used to show causal effects and datasets with more than two collection points can incorporate a meaningful temporal dimension in the analysis. Several behavioral theories posit intention as a precursor to action (Ajzen, 1985; Rogers, 1975), however, the extent, and time it takes for individuals to follow through on these intentions remains unclear. Future work should consider the pathway between intention to adapt and the actual undertaking of the action as well the temporal distance from a flood especially in underrepresented global regions.

Individual level adaption, complementing government action, is essential to address the increasing flood risk. Understanding how and why individuals adapt is critical for information transmission, motivation, and diffusion of private adaptation in societies. Culture offers a unique insight into the shared patterns of thinking and learning of individuals that can provide important context for their behavior. While culture has previously been used to explain vulnerability to disasters (Duckers et al., 2015), to our knowledge, this is the first article to statistically demonstrate the merit of including culture in climate adaptation analysis when explaining differences in the effects of factors motivating individual level behavior across an adequately large sample of countries. Researchers and policy makers can make use of these findings to better tailor their message, plan, or model and thereby more effectively motivate individual adaptation. We hope that the effect of this work will both inspire further investigation into culture (potentially on a finer scale) and motivated the inclusion of culture as variable in future disaster research.

This work was supported by the European Research Council (ERC) under the European Union's Horizon 2020 Research and Innovation Program (Grant agreement number: 758014).

\section{References}

1. Adger,W. N., Barnett, J., Brown, K., Marshall, N., and O'Brien, K. (2013). Cultural dimensions of climate change impacts and adaptation.

2. Aerts, J. C. J. H., Botzen, W. J. W., Emanuel, K., Lin, N., de Moel, H., and Michel-Kerjan, E. O. (2014). Evaluating Flood Resilience Strategies for Coastal Megacities. Science, 344(6183):473475.

3. Ajzen, I. (1985). From Intentions to Actions: A Theory of Planned Behavior. In Action Control, pages 11-39. Springer Berlin Heidelberg.

4. Bamberg, S., Masson, T., Brewitt, K., and Nemetschek, N. (2017). Threat, coping and flood prevention - A meta-analysis. Journal of Environmental Psychology.

5. Bankoff, G., Cannon, T., Kruger, F., and Schipper, L. F. (2015). Introduction: exploring the links between cultures and disasters. In Cultures and Disasters Understanding Cultural Framings in Disaster Risk Reduction, chapter Intro, pages 1-16. Routledge.

6. Barnett, J. and Breakwell, G. M. (2001). Risk Perception and Experience: Hazard Personality Profiles and Individual Differences. Risk Analysis, 21(1).

7. Berkvens, J. B. Y. (2017). The Importance of Understanding Culture When Improving Education: Learning from Cambodia. International Education Studies, 10(9):161.

8. Bin, O. and Landry, C. E. (2013). Changes in implicit flood risk premiums: Empirical evidence from the housing market. Journal of Environmental Economics and Management, 65(3):361-376.

9. Borenstein, M., Hedges, L. V., Higgins, J. P. T., and Rothstein, H. R. (2009). Introduction to Meta-Analysis. John Wiley \& Sons, Ltd, Chichester, UK.

10. Brown, C., Alexander, P., Arneth, A., Holman, I., and Rounsevell, M. (2019). Achievement of Paris 
climate goals unlikely due to time lags in the land system. Nature Climate Change, 9(3):203-208.

11. Bubeck, P., Botzen, W. J., and Aerts, J. C. (2012). A Review of Risk Perceptions and Other Factors that Influence Flood Mitigation Behavior. Risk Analysis.

12. Cannon, T. (2015). Cultures and Disasters: Understanding Cultural Framings in Disaster Risk Reduction. In Cultures and Disasters Understanding Cultural Framings in Disaster Risk Reduction, chapter 5, pages 88-106. Routledge.

13. Conway, D., Nicholls, R. J., Brown, S., Tebboth, M. G. L., Adger, W. N., Ahmad, B., Biemans, H., Crick, F., Lutz, A. F., De Campos, R. S., Said, M., Singh, C., Zaroug, M. A. H., Ludi, E., New, M., and Wester, P. (2019). The need for bottom-up assessments of climate risks and adaptation in climate-sensitive regions. Nature Climate Change, 9(7):503-511.

14. Duckers, M., Frerks, G., and Birkmann, J. (2015). Exploring the plexus of context and consequences: An empirical test of a theory of disaster vulnerability. International Journal of Disaster Risk Reduction, 13:85-95.

15. Eiser, R., Bostrom, A., Burton, I., Johnston, D. M., McClure, J., Paton, D., van der Pligt, J., and White, M. P. (2012). Risk interpretation and action: A conceptual framework for responses to natural hazards. International Journal of Disaster Risk Reduction, 1:5-16.

16. Gardoni, P. and Murphy, C. (2010). Gauging the societal impacts of natural disasters using a capability approach. Disasters.

17. GLOBE (2019). GLOBE Project. https://globeproject.com/.

18. Hofstede, G. (2019). Country Comparison HofstedeInsights.

www.hofstedeinsights.com/countrycomparison/.

19. Hofstede, G., Hofstede, G., and Minkov, M. (2010). Cultures and organizations. Intercultural cooperation and its importance for survival. McGraw Hill Publishing Company.

20. Inglehart, R., Haerpfer, C., Moreno, A., Welzel, C., Kizilova, K., Diez-Medrano, J., Lagos, M., Norris, P., Ponarin, E., Puranen, B., and Et.al. (2014). World Values Survey.

21. Jeffreys, H. (1998). Theory of probability. Clarendon Press.

22. Koerth, J., Vafeidis, A. T., and Hinkel, J. (2017). Household-Level Coastal Adaptation and Its Drivers: A Systematic Case Study Review. Risk Analysis.

23. Kruger, F., Bankoff, G., Cannon, T., Orlowski, B., and Schipper, E. L. F. (2015). Cultures and disasters: Understanding cultural framings in disaster risk reduction. Routledge.

24. Osberghaus, D. (2017). The effect of flood experience on household mitigation-Evidence from longitudinal and insurance data. Global Environmental Change.
25. Poortinga, W., Whitmarsh, L., Steg, L., B“ohm, G., and Fisher, S. (2019). Climate change perceptions and their individual-level determinants: A cross-European analysis. Global Environmental Change.

26. Rogers, R. W. (1975). A Protection Motivation Theory of Fear Appeals and Attitude Change1. The Journal of Psychology, 91(1):93-114.

27. Smith, P., Bond, M., and Kagitcibasi, C. (2006). Understanding Social Psychology Across Cultures: Living and Working in a Changing World. Sage. 15

28. Triandis, H. C., Bontempo, R., Betancourt, H., Bond, M., Leung, K., Brenes, A., Georgas, J., Hui, C. H., Marin, G., Setiadi, B., Sinha, J. B., Verma, J., Spangenberg, J., Touzard, H., and de Montmollin, G. (1986). The measurement of the etic aspects of individualism and collectivism across cultures. Australian Journal of Psychology.

29. Van Valkengoed, A. M. and Steg, L. (2019). Meta-analyses of factors motivating climate change adaptation behaviour. Nature Climate Change.

30. Veroniki, A. A., Jackson, D., Viechtbauer, W., Bender, R., Bowden, J., Knapp, G., Kuss, O., Higgins, J. P., Langan, D., and Salanti, G. (2016). Methods to estimate the between-study variance and its uncertainty in meta-analysis. Research Synthesis Methods.

31. Viechtbauer, W. (2015). Conducting MetaAnalyses in R with the metafor Package . Journal of Statistical Software.

32. Wachinger, G., Renn, O., Begg, C., and Kuhlicke, C. (2013). The Risk Perception Paradox- Implications for Governance and Communication of Natural Hazards. Risk Analysis, 33(6):1049-1065. 